\title{
Quality of Virtual Enterprise Reference Models
}

\author{
Peter Bernus
}

Griffith University, Australia, p.bernus@cit.gu.edu.au

\begin{abstract}
The article describes the need for high quality reference models for Virtual Enterprises that will speed up the creation of global enterprise networks, virtual project enterprises, and service enterprises. While many models have been presented in the literature the quality of these models has not been thoroughly researched. This article addresses the need to develop a set of design principles, and presents some examples of these, through which the usability and longevity of reference models can be improved.
\end{abstract}

\section{INTRODUCTION}

Different authors have defined the term 'Virtual Enterprise' in slightly different ways. However, a common element of these definitions is that the entity called 'Virtual Enterprise' (VE) is not an incorporated legal entity; rather it is a suitably formed joint undertaking (of shorter or longer life-span) to satisfy some business objective. It is called an enterprise because it has business objectives and processes to achieve these objectives, and it appears to be managed as one entity so as to ensure that the performance of business processes indeed takes place and those business objectives are attained.

A VE does not own (in the conventional sense) any resources nor can it be made legally responsible for its actions or the lack thereof. Yet a virtual enterprise, for the purposes of a business objective (to produce some services or goods) is behaving as if it were an incorporated legal entity and a very efficient one at that.

With the above exposition it is clear that no conventional business in its right mind would like to deal with such an elusive business entity, one that cannot be held responsible, and has no assets to back its commitments. 
Some questions that one might ask:

1. Why is it that the concept of VE is becoming so popular, and how should conventional business relate to VEs?

2. Is there any 'interface' between 'conventional' and 'virtual' enterprises?

3. Are there any virtual enterprises, which are dissimilar in some significant properties from conventional businesses that established integrated information flow between the participants?

4. Is it possible that what is becoming popular today is just a redressed conventional business, somehow the term 'Virtual' tagged onto it so as to advertise its progressive nature? - Certainly there are a number of known examples of relatively conventional supply chains, which, however, through integrated information flow, are faster, operate with less overhead and exhibit very flexible behaviour through dynamic co-operative planning abilities.

In this article we try to give answers to the above questions and show what principles should guide the development of 'blueprints' for VE creation. In Section 2 we investigate the difference between VE and conventional business, In Section 3 we discuss the need for Reference Models to support the fast creation of VEs, and in Section 4 we show how various proposed architectural principles help define suitable reference models.

\section{WHAT MAKES A VIRTUAL ENTERPRISE DIFFERENT FROM CONVENTIONAL BUSINESS?}

In our view the basis for an answer to the above questions is the understanding that a VE is really virtual, i.e. 'real' businesses and real customers interface with 'real' businesses, never with 'virtual' ones. It is only that the real business that faces the customer (or another business) is capable of acting as if it were a much larger business able to provide products and services and back them with the usual expected commitments that go with such a deal - commitments that only the community of real businesses that formed the virtual enterprise can in fact provide.

The above necessitates a set of commitments among these 'real' businesses, so as for any particular customer order the commitments to provide a complete service and the guarantee to support the product or follow up the service, is clearly and completely defined and project a trustworthy image.

One problem with VEs providing goods and services is that VEs as dynamic entities are designed to be 'ephemeral' in nature. VEs are created for a given purpose and dissolved once the objective has been satisfied. (This 
makes them very economical, because VEs do not have any overhead, they only dispose over resources as much as they actually need them to produce or add value.) However, from the customer's point of view when a VE designs and builds a product, this VE no longer exists during the time the product operates or when the product is maintained or decommissioned.

Thus the 'Business Model' behind a VE not only has to describe the ways of providing a market with goods and services of a certain kind, but it also must identify and describe a number of other entity types necessary to support the complete life of the products and services (including operational support and dissolving or recycling these as needed).

In order to create such a 'Business Model', one must define all involved enterprise entity types and their roles, interests and relationships within the overall value chain, and guarantee that the overall model operates as if all products and services were produced and supported as necessary for their entire life by one larger enterprise.

In addition to this ephemeral property, what makes VEs different from conventional businesses that use electronic means to integrate information flow is the extent and completeness of this integration, where this extent is measured in terms of how well this integration hides the fact that the VE achieves its mission through co-operation and co-ordination of many partners rather then through being a single large enterprise. We shall further elaborate on the required characteristics of this co-operation and coordination in Section 4.)

A common way to satisfy the above needs is to create Enterprise Networks. An Enterprise Network is an alliance of businesses formed for the exploitation of some kind of business opportunity. Participation in a Network (as well as entry and exit) is guided by contracts and agreed processes, which allow any member of the Network (according to the Network's cooperation contract) to respond to a customer order and to draw upon the resources of other members in the network. Enterprise Networks may be characterised by defining the set of functions that partners share as opposed to the set of functions that they do not share.

E.g. a network may be a sole marketing network, where partners only share marketing functions but do not share production and service delivery. Another network may share part of its logistic functions, such as transport and delivery, as well as marketing and quality control and product classification (such as in some agricultural co-operatives) but not share the production processes.

Some networks make a viable or even attractive business proposition (win-win for all parties, including the customers) and some do not (winloose or loose-loose). The 'business model' needs to be investigated from the 
point of view of value adding and gain distribution, to establish that the interest of all parties is compatible with the overall business interest.

Since a Network is a relatively stable organisation - with or without substantial resources of its own (typically without) - it is possible for the participating businesses to develop a set of contracts and processes that guide all aspects of joint co-operative action. Thus at the time a business need arises (i.e. when a customer enquiry or order is received) network partners can create a virtual enterprise on demand - or immediately.

What makes the VE different from a supply chain with integrated information flow is the nature of this information flow. While conventional businesses have been exchanging information using electronic means for decades (such as orders, delivery schedules, payments etc. or as in recently popularised B2B transactions), a VE extends this information flow to all levels of management to achieve complete co-ordination of the joint activity. Partners involved in VEs have co-ordination on the strategic, tactical and operational levels, as well as this co-ordination extends to product related and resource related management decisions.

\section{THE NEED FOR REFERENCE MODELS}

From the above considerations it is clear that to exploit the idea of the VE, today's businesses need blueprints or 'Reference Models' that describe viable building blocks and combination rules of partner-, network- and VE functions. These building blocks can then be customised and combined for a given type of network and its VEs. Thus we develop reference models of processes for network and VE creation as well as reference models of business processes that are performed by the network and its VEs.

While blueprint-type Reference Models are necessary for businesses to be able to embark on a new way of doing business, it is usually also required that there be a 'step-by-step' methodology that businesses could follow to build networks and networks can follow to build VEs.

Since a methodology in general is a collection of procedures, rules, methods and tools, it is not necessarily possible to attain this goal. However, the more specialised the objective (the more we know the intended business model and therefore the type of network we intend to create), the more chances there are that a specialised methodology can indeed be developed in form of a step-by-step procedure.

Who should develop such step-by-step methodologies? - Clearly, the research community in business management and IT/IS can only be made responsible for the development of generic methodologies, those that are not step-by-step. This is because the amount of detail and know-how that is 
needed for a step-by-step methodology is a commercial commodity, thus not likely to be published in the open literature.

Consulting companies are another possible source of such step-by-step methodologies, but the problem is that selling such a methodology cannot be repeated many times over.

End users (i.e. potential partners in Networks) would be ideal candidates to pool resources and develop their in-house methodology, but with the exception of large companies, resources for such planning and development are scarce. Even though the IT resources that are needed for forming simple VEs and networks become cheaper and more accessible, the complexity and the risk involved in designing viable and significantly new business networks and VEs limits the level of innovation that small and medium enterprises can attain.

The result of the above situation is a potential polarisation of the business world, with large companies being the only ones that can tap into the new potentials of VEs, and small companies being only followers. It is argued, that for a healthy business world the innovation capability must be preserved for small and medium enterprises in the same way as for large companies, and a second set of reference models is necessary. This second set of reference models would be ones describing how government, industry associations and small and medium sized companies may be able to exploit the techniques necessary to successfully invent new ways of doing business.

One example of a model with similar aim is the European Community's 6th Framework Programme, which takes an active role in the support of small and medium sized enterprises to enter this new era of competition. While many projects (in the precursor 5th Framework Programme) have been directed at the use of information technology (such as supporting electronic business) - with many interesting results and demonstrations - there has been a lack of strong results in terms of legal and business management, or really innovative ways of doing business. Most projects use information integration technologies to create quite usual value chains that operate faster, more reliably, with better co-ordination, or implement a business involving geographically dispersed business participants.

Results of some pre-competitive research projects - as developed in the Globemen Consortium and other IMS consortia - address this gap, especially in terms of responsibility structures for businesses, networks of businesses and virtual enterprises, as well as in terms of using the agent concept to dynamically build global enterprise entities (Bernus, Nemes, 1999). The Globemen consortium developed management models for Partners, Networks and Virtual Project Enterprises, identifying the interfaces and responsibility structures between their respective decision frameworks. These models - on the high level - describe the decision roles and frameworks for each 
of these enterprise entity types (the description is given in form of GRAI Grids). On the more detailed level the activities and information flow is described, as it occurs within and among management roles relevant to the Network and VE formation, operation and decommissioning. These more detailed descriptions are produced as IDEF0 models.

A note on the side: we differentiate between activity (functional) models (expressed as GRAI Grids, IDEF0 models, Use Case diagrams, etc), and process models (expressed in IDEF3, CIMOSA / First Step, UML collaboration diagrams, or UML sequence diagrams, etc). It has been observed in the practice of reference model development (Kalpic, Bernus, 2002) that sufficiently generic activity models can always be produced, i.e. for any level of management and for any function. In contrast, process models (behavioural models) can only be produced for such functions, which have a procedural nature, and/or only for a level of detail where generic procedures exist. Even if in many cases a behavioural model can be produced, usually this can only be done at the expense of genericity. Thus the Reference Model needs to be based on activity models, which can later be detailed using process (behavioural) models for more concrete cases. Of course for those functions that have an industry wide accepted step-by-step procedure there is no obstacle to developing behavioural descriptions as well.

The way we express these reference models for a given network type could be an example for other types of networks to describe their own reference models. The present models describe networks formed by a few larger partnering companies that co-operate in the design, procurement and construction of one-of-a-kind products as well as in after sales service. Further work includes the identification of transactions among the management roles for partners, networks and virtual project enterprises (using IDEF1X schemata and where generic procedures exist, IDEF3 process diagrams). Thus while lower level functions do not need to be prescribed procedurally (individual companies may follow different internal procedures), the interenterprise transactions will be described by process models and accompanying information models defining the information content of messages in these transactions.

Note that a similar approach is followed in B2B standardisation efforts, such as Rosettanet; however, these B2B models start with process models, where business to business transactions are expressed in form of behavioural models (such as UML collaboration diagrams). This may be suitable for operational level transactions, but at all except the highest level of granularity common procedural standards are not desirable when business-to-business transactions for tactical and strategic levels are designed (such as for joint scheduling, planning and strategy making). Procedural transactions on these high levels may be restricted to 'business protocols' in the sense that transac- 
tions need to consist of propositions / counter-propositions and acceptance and delivery reports. This is because procedural standards on the lower levels of granularity (i.e. beyond defining the signatures of interacting entities) contradict the information hiding principle. The violation of this principle either forces total homogeneity on the partners, where every partner uses the same algorithms to develop joint plans and schedules - an unlikely to succeed approach -, or increases the complexity of the joint action - thus making the solution more brittle in the face of change in any of the involved parties.

\section{REFERENCE MODELS AND PRINCIPLES FOR THEIR FUTURE DEVELOPMENT}

To develop generic and practical transaction protocols between businesses one may consider the applicability of design principles developed during the past 25 years for the design of complex systems. Note that Suh (1990) has developed two axioms of design that promise to encompass all design principles in all disciplines, thus it is an interesting research question whether all necessary principles listed above are indeed consequences of these two axioms. If this is indeed the case then the relationship between Suh's axioms and the desired set of design principles as advocated in this article is the same as the relationship of the axioms of logic to the theorems of mathematics while in theory it was not necessary to know more then the axioms, this fact did not eliminate the need for Russel and Whitehead's Principia Mathematica $(1910,1912,1913)$. Specifically, principles we wish to consider should apply to enterprise entities that consist of humans and automated systems (machinery and computers). Some principles are presented below.

- Principles for the reduction of complexity in system design and eventually implementation and maintenance (one example for these principles is information hiding, application of which allows independent changes to occur in subsystems / component systems and in general prevent the proliferation of change effects);

- The principle of constructing systems of systems, where a complex system is built from less complex systems so as the apparent complexity of the resulting system does not compound the complexities of the constituents. By this we mean that without knowing the internal structure of the component systems the 'interesting' properties of the higher-level system may be derived without knowing the internal structure of the components. Of course the result of such system construction is dependent on what the interesting properties of the higherlevel system are deemed to be. E.g. in constructing audio systems us- 
ing operational amplifiers the knowledge of the actual circuitry of the components is unnecessary, because for the derivation of the behaviour of the complete system we only need to know the transfer function of the involved operational amplifiers (and some operational boundaries);

- Construction principles of human / socio-technical systems, as derived by behavioural scientists, ergonomists, sociographers, organisational psychologists and management scientists. E.g. humans need to receive positive reinforcements, motivation, and a sense of progress to perform at the best of their abilities; humans and organisations in general need stretches of 'secure' times to be able to concentrate on the job at hand; conflicts of interest arise when decisional powers are distributed in certain undesirable ways as humans are unable to completely disassociate themselves from playing multiple contradicting roles, etc;

- Good designs are orthogonalised, meaning that the designed entity's function is specified as a combination or interaction of independent (or orthogonal) functions. This ensures that even if not at all possible combined functionality has been specified at the outset, the system is capable of being extended and developed at minimal cost since new combinations do not need to change the constituent functions (and their implementing modules) - only new combinations need to be implemented, or simply configured.

This list is not exhaustive but we tried to list some of the most important principles to be able to demonstrate how they apply to the design of suitable reference models for partner companies, networks and VEs. We should list many more principles, applicable to specific technical domains, such as the layering principle of computer infrastructure services, even though most are in fact specific cases of the above more generic principles.

One such generic reference model that could satisfy the above criteria is the 'multi-agent' model. We refer to agents as defined by the Artificial Intelligence community, more specifically the 'distributed $\mathrm{AI}$ ' community (Wooldridge, Jennings, 1995; Barbuceanu, Teigen, 1998). Thus an agent for our purposes is an aware agent, a) with objectives, b) autonomously acting using its own resources, c) with the (apparent) ability to plan its actions, d) observing its own progress towards the objective, e) taking remedial action if necessary, and f) interacting with its environment that may contain other agents. In addition to this basic definition, distributed AI agents have the ability to g) reason about and negotiate with other agents to agree on joint objectives and joint action (plans and schedules), h) apply the same ability as (d) and (e) onto the joint action. In distributed AI many additional properties are defined, depending on which the agent may have additional desirable 
properties, but for the purposes of this discussion we only require the above basic characteristics.

While this model has been developed for the purposes of understanding how artificial agents may be created and aggregated, it is important to note that such properties are the most desirable properties of any enterprise entity, be it a company, a network of companies, a project or a complex product (system) comprised out of either humans or machines or both. After all the aim of VE design is to create enterprises (virtual ones and real ones) so that the enterprise acting in a negotiated co-operation with other enterprises could achieve a joint objective, while satisfying its own objectives, and if its actions do not contribute to the progress any more it should be aware of this and take remedy. We call the enterprise that behaves as an agent an aware enterprise.

A further characteristic of some distributed AI systems is that agent negotiation for joint action always follows the same negotiation protocol. While this is not a priori necessary, some models stipulate such a selfsimilarity property. For discussion of such models see Warnecke's 'Fractal Factory' (Warnecke, 1993).

General Systems Theory (von Bertalannfy, 1968) describes systems as processes in dynamic equilibriums with their environment, and Holonic Systems (Koestler, 1968; Tharumarajah et al, 1996) describe systems of autonomous entities forming higher-level autonomous entities (Holons), placing further constraints on what desirable designs we must encapsulate in the Virtual Enterprise Reference Model.

The Globemen consortium VE Reference Models - in their state, as of this writing (March, 2002) - define functional requirements of the management structure of partners, networks and Virtual Project Enterprises. The coordination links between these entities (and among their constituent decision centres, or management roles) define the user requirements of such systems. The Globemen Management model and Functional Model (Olegario, 2000; Tolle, et al, 2002] describe the necessary management and control functions and interfaces.

The next step in the development of a high quality reference model would have to be the application of the listed principles to this user requirements model. The resulting model could be called a system requirements model in which all desired user requirements are satisfied, but in addition to that the model would display the properties of orthogonality, self-similarity and holonic autonomy, and each interface - as identified in the user requirements model - would be defined on the basis of agent negotiation protocols, including its behaviour in terms of transactional processes (Petri Nets, CIMOSA, FirstSTEP, UML sequence diagrams or IDEF3 process models) and state (IDEF3 Object State Transition diagrams, state-charts, or Coloured 
Petri Nets, etc), but trying to hide as much as possible from the internal state and behaviour of the negotiating entities, so as to satisfy the complexity reduction and information hiding principle. Still the contents (the messages) in these protocols would have to be defined one by one, using traditional data modelling techniques.

Finally we address an important quality of enterprise models, which have a strong indirect influence on the characteristics of the technical and human systems built on the basis of these models. It is widely believed that completeness and consistency are indispensable qualities of enterprise models. However, it is not elaborated in detail what we mean by these terms, nor is it specifically stated what level of completeness and consistency is required from enterprise models. To investigate this issue it is helpful to consider the pragmatic use of models and define completeness and consistency in terms of what is required from these models when people use them or machines process them.

As has been discussed in (Bernus et al, 1996) there is a marked difference between mathematical, or formal, completeness and consistency and pragmatic completeness and consistency. The former applies to machine processed models, while the second applies to all models. Thus, for example, formal models alone are inadequate if used in isolation, because only a subset of the pragmatic uses is satisfied by their being complete mathematical models. Formal models (where completeness entails the ability to automatically analyse and execute them to derive desired properties of the entity modelled) are desirable in case of Generic Enterprise Models (IFIP-IFAC Task Force, 1999; ISO 15704-2000, 2000) whereby ontological theories allow the precise definition of the semantics of modelling languages use to represent enterprise models. However, formal completeness is (perhaps surprisingly) not a sufficient condition for pragmatic completeness. Reference Models to be pragmatically complete, they have to have the quality of understandability and uniform interpretability by humans, and not just humans in general, but by the population of humans who need to use and reuse these models. Bernus et al (1996) have discussed what measures one can take to ensure pragmatic completeness. If these are not considered (even in case of the availability of formal models), Reference Models will not be able to be utilised effectively for designing and building Virtual Enterprises.

\section{5}

\section{CONCLUSION}

The holonic manufacturing community develops technology usable for the detailed design and implementation of the Globemen model, i.e. based on distributed AI principles. The B2B community develops specialised 
transactions that could populate the high level Globemen models with specialised content, but would have to be enveloped in agent negotiation protocols, otherwise the 'standards' (proliferating at an alarming speed) would create a jungle much as what the programming language community has suffered from for three decades.

Further research is needed to ensure that the application of humanistic principles (third bullet point in the list) get intrinsically built into the architectural (preliminary) design phase when functions get aggregated into 'enterprise modules' ('CIMOSA functional entities' (Vernadat, 1996)) - i.e. humans, groups of humans, machines and complex automated systems, as well as complex human-machine systems.

This article placed emphasis on the need for reference models, but not just any reference model: the ones we are looking for must satisfy a number of design principles. The challenge is to bring together the various communities behind these principles; otherwise we shall end up with many competing and still unsatisfactory solutions. Finally, the concept of completeness and consistency of reference models were discussed (both formal and pragmatic) as conditions of their usability.

\section{REFERENCES}

Barbuceanu, M. Teigen, R. (1998), System Integration through Agent Coordination, in P. Bernus, K. Mertins and G. Schmidt (Eds.) Handbook on Architectures of Information Systems, Springer-Verlag.

Bernus, P. Nemes. L. (1999), Organisational Design: Dynamically Creating and Sustaining Integrated Virtual Enterprises, Proc IFAC World Congress, Han-Fu Chen, Dia-Zhan Cheng and Ji-Feng Zhang (Eds.) Vol. A, Elsevier.

Bernus, P. Nemes, L. Morris, B. (1996), The meaning of an enterprise model, in P. Bernus, L. Nemes (Eds.), Modelling and Methodologies for Enterprise Integration, Chapman and Hall, London pp 183-200.

Bertalanffy, L. v. (1968), General systems theory: Foundations, development, applications. Braziller, New York.

Doumeingts, G. Vallespir, B., Chen, D. (1998), GRAI Grid Decisional Modeling, in Handbook on Architectures of Information Systems, Springer-Verlag.

Hatvany, J. (1985), Intelligence and Cooperation in Heterarchic Manufacturing Systems, Robotics \& Computer-Integrated Manufacturing, 2(2).

IFIP-IFAC Task Force (1999), The Generalised Enterprise Reference Architecture and Methodology (GERAM) V1.6.3. http://www.cit.gu.edu.au/ bernus

ISO 15704 (2000), Requirements for Generalised Enterprise Reference Architectures and Methodologies, TC 184 SC5/WG1

Kalpic, B. Bernus, P. (2002), Reference Models of a Project Enterprise, Int. J Technology Mgmt (submitted)

Koestler, A. (1968), The Ghost in the Machine, The Macmillan Company. 
Olegario, C. (2001), A Partial Enterprise Model for the Management and Control in an Extended Enterprise Scenario, Masters Dissertation, School of CIT, Brisbane: Griffith University.

Suh, N. P. (1990), The Principles of Design, Oxford University Press.

Tharumarajah, A. Wells, A.J. Nemes, L. (1996), Comparison of the bionic, fractal and holonic manufacturing system concepts, in International Journal of CIM, 9(3).

Tølle, M. Bernus, P. Vesterager, J. (2002), Reference Models for Virtual Enterprises, Proc. PRO-VE'02, Kluwer.

Vernadat, F.B. (1996), Enterprise Modelling and Integration: Principles and Applications, London: Chapman \& Hall.

Vesterager, J. Bernus, P. Larsen, L. B. Pedersen, J. D. Tølle, M. (2001), Use of GERAM as Basis for a Virtual Enterprise Framework Model, in J. Mo and L. Nemes (Eds.), Global Engineering, Manufacturing and Enterprise Networks, Kluwer.

Whitehead, A.N. and Russell, B. $(1910,1912,1913)$ Principia Mathematica, 3 Vols, Cambridge University Press.

Warnecke, H. J. (1993), The Fractal Company: A Revolution in Corporate Culture, SpringerVerlag.

Wooldridge, M., Jennings, N. (1995), Intelligent Agents: Theory and Practice. The Knowledge Engineering Review 10(2). 